\title{
LIMIT CYCLES OF A SECOND-ORDER DIFFERENTIAL EQUATION
}

\author{
TING CHEN ${ }^{1,2}$ AND JAUME LLIBRE ${ }^{2, *}$
}

\begin{abstract}
We provide an upper for the maximum number of limit cycles bifurcating from the periodic solutions of $\ddot{x}+x=0$, when we perturb this system as follows

$$
\ddot{x}+\varepsilon\left(1+\cos ^{m} \theta\right) Q(x, y)+x=0,
$$

where $\varepsilon>0$ is a small parameter, $m$ is an arbitrary non-negative integer, $Q(x, y)$ is a polynomial of degree $n$ and $\theta=\arctan (y / x)$. The main tool used for proving our results is the averaging theory.
\end{abstract}

\section{INTRODUCTION}

To determine the number of limit cycles of a differential equation is one of the main problems in the qualitative theory of planar differential system. In 1881 Poincaré [6] defined the notion of limit cycle of a planar differential system as a periodic orbit isolated in the set of all periodic orbits of the differential system. And he defined the notion of a center of a real planar differential system, i.e. of an isolated equilibrium point having a neighborhood filled with periodic orbits. Later on one way to produce limit cycles is by perturbing the periodic orbits of a center, see for instance the papers $[7,2,4]$ and the references quoted there.

In [5] Mathieu consider the second order differential equation

$$
\ddot{x}+b(1+\cos t) x=0,
$$

where $b$ is a real constant. It is called Mathieu equation, which is the simplest mathematical model of an excited system depending on a parameter. The more general Emarkov-Pinney equation is Mathieu-Duffing type equatoins

$$
\ddot{x}+b(1+\cos t) x-x^{\beta}=0,
$$

where $\beta$ is an integer and $b>0$. These equations describe the dynamic of a system with harmonic parametric excitation and a nonlinear term corresponding to a restoring force, see the papers $[9,10,3,8]$.

We shall study the limit cycles of a kind of generalization of the second-order differential equations (1) and (2). More precisely, the objective of this paper is to consider the second-order differential equations

$$
\ddot{x}+\varepsilon\left(1+\cos ^{m} \theta\right) Q(x, y)+x=0,
$$

\footnotetext{
${ }^{0}$ Corresponding author

2010 Mathematics Subject Classification. Primary: 34C07, 34C08.

Key words and phrases. Mathieu-Duffing type, Limit cycle, Averaging theory.
} 
or equivalently its differential system of first order

$$
\begin{aligned}
& \dot{x}=y, \\
& \dot{y}=-x-\varepsilon\left(1+\cos ^{m} \theta\right) Q(x, y) .
\end{aligned}
$$

We study the maximum number of limit cycles which can bifurcate from the center of system (3) with $\varepsilon=0$, where $\varepsilon$ is sufficiently small and $\theta=\arctan (y / x)$. More precisely, we consider the planar vector field

$$
\chi=\chi(x, y)=(y,-x),
$$

and we perturb this vector field $\chi$ as follows

$$
\chi_{\varepsilon}=\chi(x, y)+\varepsilon\left(1+\cos ^{m} \theta\right)(0, Q(x, y)) .
$$

The main result of this paper is the following. For a definition of averaged function of first order see section 2 and [4].

Theorem 1. Assume that the average function $f(r)$ of first order associated to the vector field $\chi_{\varepsilon}$ is non-zero and $\varepsilon>0$ sufficiently small.

(a) If $m$ is odd, the maximum number of limit cycles of $\chi_{\varepsilon}$ bifurcating from the periodic solutions of the center $\chi$, is at most $n-1$ using the averaging theory of first order.

(b) If $m$ is even, the maximum number of limit cycles of $\chi_{\varepsilon}$ bifurcating from the periodic solutions of center $\chi$, is at most $n-1$ or $n-2$, when $n$ is odd or even, respectively.

Moreover these upper bounds are reached.

Theorem 1 is proved in section 3. Note that the maximum number of limit cycles stated in Theorem 1 depend on the numbers $m$ and $n$.

We provide a summary about the averaging theory for computing periodic solutions of vector fields that we shall use for proving Theorem 1 in section 2 .

\section{Averaging theORY FOR DIFFERENTIAL SYSTEMS}

In this section we recall some known results of the averaging theory that we shall need for proving Theorem 1. For more details on the averaging theory see [4].

Consider a non-autonomous differential equation of the form

$$
\frac{d r}{d \theta}=\chi(r, \theta)=\varepsilon F(r, \theta)+\varepsilon^{2} R(r, \theta, \varepsilon),
$$

where $r \in \mathbb{R}, \theta \in \mathbb{S}^{1}=\mathbb{R} /(2 \pi \mathbb{Z})$. And $F: D \times \mathbb{S}^{1} \rightarrow \mathbb{R}^{2}, R: D \times \mathbb{S}^{1} \times\left(-\varepsilon_{0}, \varepsilon_{0}\right) \rightarrow \mathbb{R}^{2}$ are $\mathbb{C}^{2}$ functions, $2 \pi$-periodic in the variable $\theta$ and $D$ is an open interval of $\mathbb{R}$.

The averaged function $f: D \rightarrow \mathbb{R}$ associated to system (4) is defined by

$$
f(r)=\frac{1}{2 \pi} \int_{0}^{2 \pi} F(r, \theta) d \theta .
$$

We called that if $r\left(r_{0}, \theta\right)$ is the solution of the vector field $\chi(r, \theta)$ such that $r\left(r_{0}, 0\right)=$ $r_{0}$, then we have

$$
r\left(r_{0}, 2 \pi\right)-r_{0}=\varepsilon f(r)+O\left(\varepsilon^{2}\right) .
$$


So for $\varepsilon>0$ sufficiently small the simple zeros of the averaged function $f(r)$ provides limit cycles of vector field $\chi(r, \theta)$. In order to study the simple zeros of the function $f(r)$ we shall apply the Descartes Theorem (for a proof see for instance [1]).

Descartes Theorem. Consider the real polynomial $p(r)=a_{i_{1}} r^{i_{1}}+a_{i_{2}} r^{i_{2}}+\cdots+$ $a_{i_{n}} r^{i_{n}}$ with $0 \leq i_{1}<i_{2}<\cdots<i_{n}$ and $a_{i_{j}} \neq 0$ real constants for $j \in\{1,2, \ldots, n\}$. When $a_{i_{j}} a_{i_{j+1}}<0$, we say that $a_{i_{j}}$ and $a_{i_{j+1}}$ have a variation of sign. If the number of variations of signs in $m$, then $p(r)$ has at most $m$ positive real roots. Moreover, it is always possible to choose the coefficients of $p(r)$ in such a way that $p(r)$ has exactly $n-1$ positive real roots.

\section{Proof of Theorem 1}

Assume that the polynomial $Q(x, y)=\sum_{i+j=0}^{n} a_{i j} x^{i} y^{j}$. Doing the change of variable $(x, y) \rightarrow(r, \theta)$, where $x=r \cos \theta, y=r \sin \theta$, with $r>0$, system (3) associated to the vector field $\chi_{\varepsilon}$ in coordinates $(r, \theta)$ writes

$$
\begin{aligned}
& \dot{r}=-\varepsilon \sum_{i+j=0}^{n} R_{i j}(\theta) r^{i+j}, \\
& \dot{\theta}=-1-\varepsilon \sum_{i+j=0}^{n} \Theta_{i j}(\theta) r^{i+j-1},
\end{aligned}
$$

where

$$
\begin{aligned}
& R_{i j}(\theta)=a_{i j}\left(\cos ^{i} \theta \sin ^{j+1} \theta+\cos ^{i+m} \theta \sin ^{j+1} \theta\right), \\
& \Theta_{i j}(\theta)=a_{i j}\left(\cos ^{i+1} \theta \sin ^{j} \theta+\cos ^{i+m+1} \theta \sin ^{j} \theta\right) .
\end{aligned}
$$

Taking $\theta$ as the new independent variable the previous differential system becomes the differential equation

$$
\frac{d r}{d \theta}=\varepsilon \sum_{i+j=0}^{n} R_{i j}(\theta) r^{i+j}+O\left(\varepsilon^{2}\right)=\varepsilon F(r, \theta)+O\left(\varepsilon^{2}\right) .
$$

Note that this differential equation is written in the normal form (4) for applying the averaging theory of first order.

In our study we will use the following formulas for compute the averaged function. The first formula is

$$
\int_{0}^{2 \pi} \cos ^{p} \theta \sin ^{2 q} \theta d \theta=\frac{(2 q-1) ! !}{(2 q+p)(2 q+p-2) \cdots(p+2)} \int_{0}^{2 \pi} \cos ^{p} \theta d \theta,
$$

for more details of this integral and the other ones integral see pages 152-153 of [11]. This formula is applicable for arbitrary real $p$ and arbitrary positive integer $q$, except for the following negative even integers $p=-2,-4, \ldots,-2 n$. If $p$ is a natural integer and $q=0$, we have the formulas

$$
\begin{aligned}
& \int_{0}^{2 \pi} \cos ^{2 l} \theta d \theta=\frac{(2 l-1) ! !}{2^{l} l !} 2 \pi \\
& \int_{0}^{2 \pi} \cos ^{2 l+1} \theta d \theta=0 .
\end{aligned}
$$


We also have the formula

$$
\int_{0}^{2 \pi} \cos ^{p} \theta \sin ^{2 q+1} \theta d \theta=0 .
$$

This last formula is applicable for arbitrary real $p$ and non-negative integer $q$, except for the following negative odd integers $p=-1,-3, \ldots,-(2 n+1)$. Therefore, from the previous section, we consider two cases and some subcases in order to study the averaged function associated to the differential equation (5).

Case (a). Let $m$ be an odd. Then we consider two subcases for studying the averaged function.

Subcase (a.1). If $n$ is an even, we have

$$
\begin{aligned}
& f_{1}(r)=\frac{1}{2 \pi} \int_{0}^{2 \pi} F(r, \theta) d \theta \\
& =\frac{1}{2 \pi} \int_{0}^{2 \pi} \sum_{i+j=0}^{n}\left[a_{i j}\left(\cos ^{i} \theta+\cos ^{i+m} \theta\right) \sin ^{j+1} \theta\right] r^{i+j} d \theta \\
& =\frac{1}{2 \pi} \int_{0}^{2 \pi} \sum_{i+2 q=2}^{n+1}\left[a_{i, 2 q-1}\left(\cos ^{i} \theta+\cos ^{i+m} \theta\right) \sin ^{2 q} \theta\right] r^{i+2 q-1} d \theta \\
& =\frac{1}{2 \pi}\left[\sum_{2 l+1+2 q=3}^{n+1} a_{2 l+1,2 q-1} r^{2 l+2 q} \int_{0}^{2 \pi} \cos ^{2 l+m+1} \theta \sin ^{2 q} \theta d \theta\right. \\
& \left.+\sum_{2 l+2 q=2}^{n} a_{2 l, 2 q-1} r^{2 l+2 q-1} \int_{0}^{2 \pi} \cos ^{2 l} \theta \sin ^{2 q} \theta d \theta\right] \\
& =\sum_{l+q=1}^{n / 2} a_{2 l+1,2 q-1} r^{2 l+2 q} \frac{(2 q-1) ! !}{(2 q+2 l+1)(2 q+2 l-1) \cdots(2 l+3)} \frac{(2 l+m) ! !}{2^{\frac{2 l+m+1}{2}}\left(\frac{2 l+m+1}{2}\right) !} \\
& +\sum_{l+q=1}^{n / 2} a_{2 l, 2 q-1} r^{2 l+2 q-1} \frac{(2 q-1) ! !}{(2 q+2 l)(2 q+2 l-2) \cdots(2 l+2)} \frac{(2 l-1) ! !}{2^{l} l !} \\
& =\sum_{l+q=1}^{n / 2} a_{2 l+1,2 q-1} r^{2 l+2 q} \frac{(2 l+m) ! !(2 q-1) ! !}{2^{\frac{2 l+m+1}{2}}\left(\frac{2 l+m+1}{2}\right) !(2 q+2 l+1)(2 q+2 l-1) \cdots(2 l+3)} \\
& +\sum_{l+q=1}^{n / 2} a_{2 l, 2 q-1} r^{2 l+2 q-1} \frac{(2 l-1) ! !(2 q-1) ! !}{2^{l+q} l !(q+l)(q+l-1) \cdots(l+1)} \\
& =\sum_{k=1}^{n} A_{k} r^{k}
\end{aligned}
$$


Subcase (a.2). If $n$ is an odd, we have

$$
\begin{aligned}
f_{2}(r)= & \frac{1}{2 \pi} \int_{0}^{2 \pi} F(r, \theta) d \theta \\
= & \frac{1}{2 \pi} \int_{0}^{2 \pi} \sum_{i+j=0}^{n}\left[a_{i j}\left(\cos ^{i} \theta+\cos ^{i+m} \theta\right) \sin ^{j+1} \theta\right] r^{i+j} d \theta \\
= & \frac{1}{2 \pi} \int_{0}^{2 \pi} \sum_{i+2 q=2}^{n+1}\left[a_{i, 2 q-1}\left(\cos ^{i} \theta+\cos ^{i+m} \theta\right) \sin ^{2 q} \theta\right] r^{i+2 q-1} d \theta \\
= & \frac{1}{2 \pi}\left[\sum_{2 l+1+2 q=3}^{n} a_{2 l+1,2 q-1} r^{2 l+2 q} \int_{0}^{2 \pi} \cos ^{2 l+m+1} \theta \sin ^{2 q} \theta d \theta\right. \\
& \left.+\sum_{n+1}^{n+1} a_{2 l, 2 q-1} r^{2 l+2 q-1} \int_{0}^{2 \pi} \cos ^{2 l} \theta \sin ^{2 q} \theta d \theta\right] \\
= & \sum_{l+q=1}^{2 l+2 q=2} a_{2 l+1,2 q-1} r^{2 l+2 q} \frac{(2 l+m) ! !(2 q-1) ! !}{2^{\frac{2 l+m+1}{2}}\left(\frac{2 l+m+1}{2}\right) !(2 q+2 l+1)(2 q+2 l-1) \cdots(2 l+3)} \\
& +\sum_{l+q=1}^{(n+1) / 2} a_{2 l, 2 q-1} r^{2 l+2 q-1} \frac{(2 l-1) ! !(2 q-1) ! !}{2^{l+q} l !(q+l)(q+l-1) \cdots(l+1)} \\
= & \sum_{k=1}^{n} \widetilde{A}_{k} r^{k} .
\end{aligned}
$$

Case (b). Assume $m$ is an even, we consider the following subcases for studying the averaged function.

Subcase (b.1). If $n$ is an even, we have

$$
\begin{aligned}
f_{3}(r)= & \frac{1}{2 \pi} \int_{0}^{2 \pi} F(r, \theta) d \theta \\
= & \frac{1}{2 \pi} \int_{0}^{2 \pi} \sum_{i+j=0}^{n}\left[a_{i j}\left(\cos ^{i} \theta+\cos ^{i+m} \theta\right) \sin ^{j+1} \theta\right] r^{i+j} d \theta \\
= & \frac{1}{2 \pi} \int_{0}^{2 \pi} \sum_{i+2 q=2}^{n+1}\left[a_{i, 2 q-1}\left(\cos ^{i} \theta+\cos ^{i+m} \theta\right) \sin ^{2 q} \theta\right] r^{i+2 q-1} d \theta \\
= & \frac{1}{2 \pi}\left[\sum_{2 l+2 q=2}^{n} a_{2 l, 2 q-1} r^{2 l+2 q-1} \int_{0}^{2 \pi}\left(\cos ^{2 l} \theta+\cos ^{2 l+m} \theta\right) \sin ^{2 q} \theta d \theta\right] \\
= & \sum_{l+q=1}^{n / 2} a_{2 l, 2 q-1} r^{2 l+2 q-1} \frac{(2 q-1) ! !}{2^{q}(q+l)(q+l-1) \cdots(l+1)}\left[\frac{(2 l-1) ! !}{2^{l} l !}\right. \\
& \left.+\frac{(2 l+m-1) ! !}{2^{\frac{2 l+m}{2}}\left(\frac{2 l+m}{2}\right) !}\right] \\
= & \sum_{k=1}^{n-1} B_{k} r^{k} .
\end{aligned}
$$


Subcase (b.2). If $n$ is an odd, we have

$$
\begin{aligned}
f_{4}(r)= & \frac{1}{2 \pi} \int_{0}^{2 \pi} F(r, \theta) d \theta \\
= & \frac{1}{2 \pi} \int_{0}^{2 \pi} \sum_{i+j=0}^{n}\left[a_{i j}\left(\cos ^{i} \theta+\cos ^{i+m} \theta\right) \sin ^{j+1} \theta\right] r^{i+j} d \theta \\
= & \frac{1}{2 \pi} \int_{0}^{2 \pi} \sum_{i+2 q=2}^{n+1}\left[a_{i, 2 q-1}\left(\cos ^{i} \theta+\cos ^{i+m} \theta\right) \sin ^{2 q} \theta\right] r^{i+2 q-1} d \theta \\
= & \frac{1}{2 \pi}\left[\sum_{2 l+2 q=2}^{n+1} a_{2 l, 2 q-1} r^{2 l+2 q-1} \int_{0}^{2 \pi}\left(\cos ^{2 l} \theta+\cos ^{2 l+m} \theta\right) \sin ^{2 q} \theta d \theta\right] \\
= & \sum_{l+q=1}^{(n+1) / 2} a_{2 l, 2 q-1} r^{2 l+2 q-1} \frac{(2 q-1) ! !}{2^{q}(q+l)(q+l-1) \cdots(l+1)}\left[\frac{(2 l-1) ! !}{2^{l} l !}\right. \\
& \left.+\frac{(2 l+m-1) ! !}{2^{\frac{2 l+m}{2}}\left(\frac{2 l+m}{2}\right) !}\right] \\
= & \sum_{k=1}^{n} B_{k} r^{k} .
\end{aligned}
$$

Note that the coefficients $a_{i j}$ of the vector field $\chi_{\varepsilon}$ which appear in $A_{k}$ (or $\widetilde{A}_{k}$, or $B_{k}$ ) can be arbitrarily chosen. From the above expressions of the four subcases we obtain that the averaged function $f(r)$ is generated by a linear combination of a set $\mathcal{F}_{l}=\left\{r, r^{2} \ldots, r^{l}\right\}$ with $l \in\{n-1, n\}$. Using the Descartes Theorem, it follows that $f(r)$ can have at most $n-1$ (or at most $n-2$ when $n$ and $m$ are even numbers) simple zeros. Therefore for $\varepsilon>0$ sufficiently small the vector field $\chi_{\varepsilon}$ can have at most $n-1$ (or $n-2$ ) limit cycles. This completes the proof of Theorem 1 .

\section{ACKNOWLEDGMENTS}

The first author is partially supported by National Natural Science Foundation of China (No. 11771059).

The second author is partially supported by the MINECO grants MTM201340998-P and MTM2016-77278-P (FEDER) and the AGAUR grant 2014 SGR568.

\section{REFERENCES}

[1] I.S. Berezin, N.P. Zhidkov, Computing Methods, vol. II, Pergamon Press, Oxford, 1964.

[2] A. Buică, J. Giné, J. Llibre, Bifurcation of limit cycles from a polynomial degenerate center, Adv. Nonlinear Stud. 10 (2010), 597-609.

[3] E. Esmailzadeh, G. Nakhaie-Jazar, Periodic solutions of a Mathieu-Duffing type equation, J. Non. Linear Mech. 32 (1997), 905-912.

[4] J. Llibre, R. Moeckel, C. Simó, Central Configurations, Periodic orbits and Hamiltonian systems, Advanced Courses in Mathematics CRM Barcelona, Birkhäuser, 2015.

[5] É. Mathieu, Mémoire sur le mouvement vibratoire d'une membrane de forme elliptique, J. Math. Pures Appl. 13 (1868), 137-203.

[6] H. Poincaré, Mémoire sur les courbes définies par une équation différentielle I, II, J. Math. Pures Appl. 7 (1881), 375-422; 8 (1882), 251-296. 
[7] L.S. Pontrjagin, Über Autoschwingungssysteme, die den hamiltonschen nahe liegen, Phys. Z. Sowjetunion 6 (1934), 25-28.

[8] P.J. Torres, Non-trivial periodic solutions of a non-linear Hill's equation with positively homogeneous term, Nonlinear Anal. 65 (2006), 841-844.

[9] M. Zamora, A note on the periodic solutions of a Mathieu-Duffing type equations, Math. Nachr. 290 (2017). 1113-1118.

[10] M. Zhang, Periodic solutions of equations of Emarkov-Pinney type, Adv. Nonlinear Stud. 6 (2006), 57-67.

[11] D. Zwillinger, Table of Integrals, Series, and Products, ISBN: 978-0-12-384933-5 2014

1 College of Mathematics and Econometrics, Hunan University, Changsha, 410082, PR CHINA

E-mail address: chenting0715@126.com

2 Departament de Matemàtiques, Universitat Autònoma de Barcelona, 08193 Bellaterra, Barcelona, Catalonia, Spain

E-mail address: jllibre@mat.uab.cat 